\title{
Adomian decomposition method for solving initial value problems in vibration models
}

\author{
Sudi Mungkasi ${ }^{1, *}$, and I Made Wicaksana Ekaputra $^{2}$ \\ ${ }^{1}$ Department of Mathematics, Faculty of Science and Technology, Sanata Dharma University, \\ Mrican, Tromol Pos 29, Yogyakarta 55002, Indonesia \\ ${ }^{2}$ Department of Mechanical Engineering, Faculty of Science and Technology, Sanata Dharma \\ University, Mrican, Tromol Pos 29, Yogyakarta 55002, Indonesia
}

\begin{abstract}
A number of engineering problems have second-order ordinary differential equations as their mathematical models. In practice, we may have a large scale problem with a large number of degrees of freedom, which must be solved accurately. Therefore, treating the mathematical model governing the problems correctly is required in order to get an accurate solution. In this work, we use Adomian decomposition method to solve vibration models in the forms of initial value problems of secondorder ordinary differential equations. However, for problems involving an external source, the Adomian decomposition method may not lead to an accurate solution if the external source is not correctly treated. In this paper, we propose a strategy to treat the external source when we implement the Adomian decomposition method to solve initial value problems of second-order ordinary differential equations. Computational results show that our strategy is indeed effective. We obtain accurate solutions to the considered problems. Note that exact solutions are often not available, so they need to be approximated using some methods, such as the Adomian decomposition method.
\end{abstract}

\section{Introduction}

Vibration occurs in daily life, such as sounds, acoustics, machines, etc. A mathematical model for vibrations is the second-order ordinary differential equations. The model can be either with or without source terms. A source term is assumed to be an external force involved in the vibration.

The vibration model can have a high degree of freedom, so solving the model can be tedious. A number of researchers have attempted to solve vibration model, such as Nad [1], Ouyang and Zhang [2], and Supriyadi [3]. Nevertheless, it is still an open problem about how to solve the model in an inexpensive computations.

In this paper, we consider vibration models, especially in the scalar form. We use the Adomian decomposition method due to Adomian [4]. The Adomian decomposition method is chosen, as it has some advantages, such as that it is meshless, so solutions can be computed at any time [5-6]. We propose a computational treatment of the source term,

\footnotetext{
* Corresponding author: sudi@usd.ac.id
} 
when it appears in the vibration model and when we implement the Adomian decomposition method. If the source term is not appropriately treated, the method may lead to a misleading solution.

The rest of the paper is organised as follows. Section 2 provides the mathematical models we consider. Section 3 proposes the computational treatment of the source term of the vibration model. Results and discussion are given in Section 4. We conclude the paper in Section 5.

\section{Mathematical models}

We consider the vibration problem of a spring-mass system having the vector-matrix form

$$
\mathbf{M} \ddot{\mathbf{x}}+\mathbf{C} \dot{\mathbf{x}}+\mathbf{K x}=\mathbf{s}(t),
$$

where $\mathbf{x}$ is the state space vector dependent on the free variable time $t, \mathbf{M}$ is the mass matrix, $\mathbf{C}$ is the friction matrix, $\mathbf{K}$ is the stiffness matrix, and $\mathbf{s}(t)$ is the source-term vector. The source term $\mathbf{s}(t)$ is linearly independent with the $\mathbf{C} \dot{\mathbf{x}}$ and $\mathbf{K x}$ terms.

When there is no damping, the initial value problem of the vibration model in the scalar form can be written as

$$
x^{\prime \prime}(t)=k x+s(t), \quad x(0)=\alpha, \quad x^{\prime}(0)=\beta
$$

defined in a closed domain $[0, b]$. Here, $t$ is the time variable which is free, and $x$ is the position variable dependent on $t$. In addition, $\alpha$ and $\beta$ are constants, and $b$ is a positive constant. The short notation $x^{\prime}(t)$ means $d x / d t$, which is the first derivative of $x$ with respect to $t$. The short notation $x^{\prime \prime}(t)$ is for $d^{2} x / d t^{2}$, which is the second derivative of $x$ with respect to $t$. We assume that all functions involved in the model are smooth, and the source term $s(t)$ is linearly independent with the $k x$ term, and can be either linear or nonlinear. Here, $k$ is constant.

In this paper, we focus on solving the initial value problem (2) using the Adomian decomposition method. Care should be taken when we have the nonzero source term $s(t)$ in the equation. Otherwise, the Adomian decomposition method may lead to inaccurate results.

\section{Adomian decomposition method}

In this section, we provide the Adomian decomposition procedure following the work of Al-Khaled and Anwar [7]. This is the complement of the work of Biazar, Babolian, and Islam [8].

We consider the differential operator $L$ defined as $L=d^{2} / d t^{2}$. Then the inverse operator $L^{-1}$ is defined as

$$
L^{-1}=\int_{0}^{t} \int_{0}^{t}(\cdot) d t d t
$$

The initial value problem (2) can be written in an operator form as

$$
L x=k x+s(t) .
$$

Knowing the initial values $x(0)=\alpha$ and $x^{\prime}(0)=\beta$, we find that equation (4) becomes

$$
x(t)=\alpha+\beta t+L^{-1}[s(t)]+L^{-1}[k x] .
$$


The Adomian decomposition method works by assumption that the function $x(t)$ can be decomposed into a series of functions $x_{n}(t)$, that is,

$$
x(t)=\sum_{n=0}^{\infty} x_{n}(t)
$$

Substitution of equation (6) to equation (5) results in

$$
\sum_{n=0}^{\infty} x_{n}(t)=\alpha+\beta t+L^{-1}[s(t)]+L^{-1}\left[\sum_{n=0}^{\infty} k x_{n}(t)\right] .
$$

Each term of the series (7) is determined as follows

$$
\begin{gathered}
x_{0}(t)=\alpha+\beta t+L^{-1}[s(t)], \\
x_{n+1}(t)=L^{-1}\left[k x_{n}\right], \quad n \geq 0 .
\end{gathered}
$$

The nonzero source term $s(t)$ is treated in $x_{0}(t)$ of equation (8a). This treatment is important to note in order that our approximation is accurate. If the source term is treated in equation (8b) instead, then we call the treatment as a naive treatment. The $N$-term approximation of $x(t)$ is given by

$$
\phi_{N}(t)=\sum_{n=0}^{N-1} x_{n}(t) .
$$

The Adomian decomposition method is an analytical technique of approximation to a function. We do not need to discretise the given domain. The method converges to the exact solution rapidly for a certain radius of domain.

\section{Results and discussion}

In this section, we discuss our research results. As has been mentioned, we investigate the performance of the Adomian decomposition method in solving initial value problems of the second-order ordinary differential equations involving source terms. The source term in the model must be handled appropriately. If not, the Adomian decomposition method may not converge. We provide two examples in this section. These examples are extracted from the paper of Al-Khaled and Anwar [7].

\subsection{Linear source term}

As the first example, we consider the initial value problem [7] having a linear source term

$$
x^{\prime \prime}(t)=x+t, \quad x(0)=1, \quad x^{\prime}(0)=0
$$

where $t \in[0,1]$. The exact solution to this problem is $x(t)=e^{t}-t$.

The equation $x^{\prime \prime}(t)=x+t$ in problem (10) can be written in an operator form as

$$
L x=x+t,
$$

for $0<t<1$. Knowing the initial conditions $x(0)=1$ and $x^{\prime}(0)=0$, we obtain

$$
x(t)=1+L^{-1}[t]+L^{-1}[x],
$$

where $L^{-1}$ is given by equation (3). Using the Adomian decomposition, equation (12) becomes 


$$
\sum_{n=0}^{\infty} x_{n}(t)=1+\frac{t^{3}}{6}+L^{-1}\left[\sum_{n=0}^{\infty} x_{n}(t)\right] .
$$

The recursive formula for the Adomian decomposition method is

$$
\begin{gathered}
x_{0}(t)=1+\frac{t^{3}}{6}, \\
x_{n+1}(t)=L^{-1} x_{n}, \quad n \geq 0 .
\end{gathered}
$$

Computing the first three components of $\left\{x_{n}(t)\right\}$, we obtain:

$$
\begin{gathered}
x_{0}(t)=1+\frac{t^{3}}{6}, \\
x_{1}(t)=\frac{t^{2}}{2}+\frac{t^{5}}{120}, \\
x_{2}(t)=\frac{t^{4}}{24}+\frac{t^{7}}{5040} .
\end{gathered}
$$

Note that the $N$-term approximation of $x(t)$ is given by equation (9).

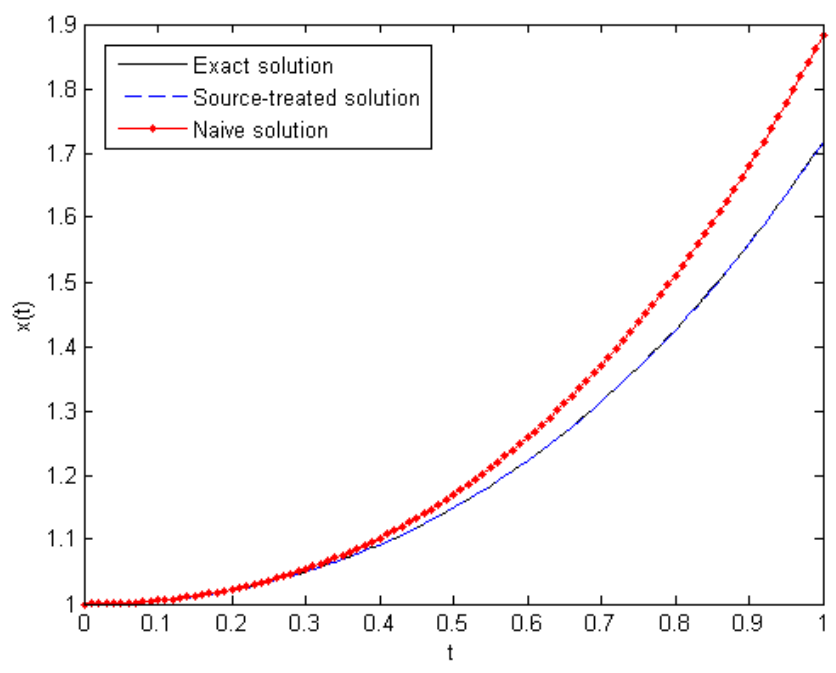

Fig. 1. Plots of the exact solution to the first example, the source-treated solution, and the naive solution. Here the source-treated and naive solutions are computed up to $x_{2}$. The exact and the source-treated solutions are almost overlapping, so we cannot see the difference between.

Figure 1 shows the curves of the exact solution to the first example, the source-treated solution, and the naive solution. We have computed the source-treated and naive solutions up to $x_{2}$. We observe that the source-treated solution, that is the solution obtained from the proposed computational treatment of the source term, is very accurate. In contrast, the naive solution is not accurate. 


\subsection{Nonlinear source term}

As the second example, we consider the initial value problem [7] having a nonlinear source term

$$
x^{\prime \prime}(t)=-\omega^{2} x+\left(\omega^{2}-1\right) \sin t, \quad x(0)=1, \quad x^{\prime}(0)=1+\omega .
$$

where $t \in[0,2 \pi]$ and $\omega>>1$. The exact solution to this problem is

$$
x(t)=\cos \omega t+\sin \omega t+\sin t .
$$

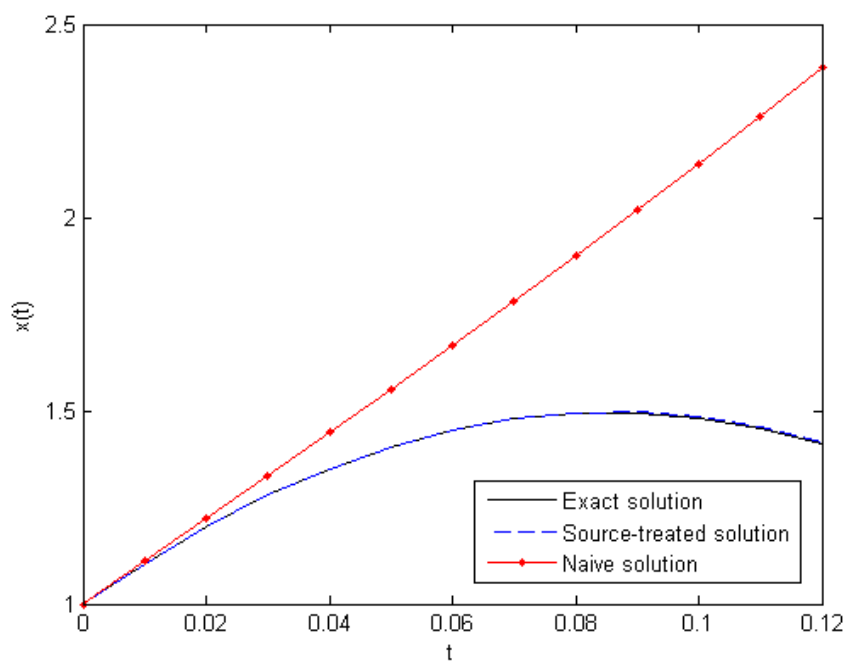

Fig. 2. Plots of the exact solution to the second example, the source-treated solution, and the naive solution. Here the source-treated and naive solutions are computed up to $x_{2}$. The exact and the source-treated solutions are almost overlapping, so we cannot see the difference.

The equation $x^{\prime \prime}(t)=-\omega^{2} x+\left(\omega^{2}-1\right) \sin t$ in problem (16) can be written in an operator form as

$$
L x=-\omega^{2} x+\left(\omega^{2}-1\right) \sin t,
$$

for $0<t<2 \pi$. Using the initial conditions $x(0)=1$ and $x^{\prime}(0)=1+\omega$, we find

$$
x(t)=1+(1+\omega) t+L^{-1}\left[\left(\omega^{2}-1\right) \sin t\right]-L^{-1}\left[\omega^{2} x\right],
$$

where, again, $L^{-1}$ is given by equation (3). Using the Adomian decomposition, equation (19) becomes

$$
\sum_{n=0}^{\infty} x_{n}(t)=1+(1+\omega) t+L^{-1}\left[\left(\omega^{2}-1\right) \sin t\right]-L^{-1}\left[\sum_{n=0}^{\infty} \omega^{2} x_{n}(t)\right] .
$$

The recursive formula for the Adomian decomposition method is

$$
\begin{gathered}
x_{0}(t)=1+(1+\omega) t+L^{-1}\left[\left(\omega^{2}-1\right) \sin t\right], \\
x_{n+1}(t)=-L^{-1}\left[\omega^{2} x_{n}\right], \quad n \geq 0 .
\end{gathered}
$$

The first three components of $\left\{x_{n}(t)\right\}$ are as follows, for $\omega=10$ : 


$$
\begin{gathered}
x_{0}(t)=1+11 t+99\left(\frac{t^{3}}{6}-\frac{t^{5}}{120}+\frac{t^{7}}{5040}\right), \\
x_{1}(t)=-100\left(\frac{1}{2} t^{2}+\frac{11}{6} t^{3}+\frac{33}{40} t^{5}-\frac{11}{560} t^{7}+\frac{11}{40320} t^{9}\right), \\
x_{2}(t)=10000\left(\frac{1}{24} t^{4}+\frac{11}{120} t^{5}+\frac{33}{560} t^{7}-\frac{11}{40320} t^{9}+\frac{1}{403200} t^{11}\right),
\end{gathered}
$$

The 3-term approximation of $x(t)$ is

$$
\phi_{3}(t)=x_{0}(t)+x_{1}(t)+x_{2}(t) .
$$

Figure 2 shows the curves of the exact solution to the second example, the sourcetreated solution, and the naive solution. Again, we have computed the source-treated and naive solutions up to $x_{2}$. This second example confirms, once again, the effectiveness of the proposed treatment of the source term in the model, when we implement the Adomian decomposition method.

\section{Conclusion}

In this paper, we have presented how the Adomian decomposition method is applied when it is used to solve second order-ordinary differential equations involving source terms. Our computational treatment of the source term is indeed effective to obtain accurate solutions to the problems. The results are limited to scalar problems. It is projected that the computational treatment will work for problems with higher degrees of freedom.

This research was financially supported by Sanata Dharma University. Both authors thank Sanata Dharma University for the financial support.

\section{References}

1. M. Nad, Structural dynamic modification of vibrating systems, Appl. Comput. Mech., 1, 203-214 (2007)

2. H. Ouyang, J. Zhang, Passive modifications for partial assignment of natural frequencies of mass-spring systems, Mech. Syst. Signal Process., 50, 214-226 (2015)

3. B. Supriyadi, Recalage des modeles de batiment (These de Doctorat, Ecole Centrale de Lyon, France, 1993)

4. G. Adomian, Solutions of nonlinear P.D.E., Appl. Math. Lett., 11, 121-123 (1998)

5. S. Mungkasi, M.F.S. Dheno, Adomian decomposition method used to solve the gravity wave equations, AIP Conf. Proc. 1788, 030103 (2017)

6. A.M. Wazwaz, Partial Differential Equations and Solitary Waves Theory (Springer, Berlin, 2009)

7. K. Al-Khaled, M.N. Anwar, Numerical comparison of methods for solving secondorder ordinary initial value problems, Appl. Math. Model., 31, 292-301 (2007)

8. J. Biazar, E. Babolian, R. Islam, Solution of the system of ordinary differential equations by Adomian decomposition method, Appl. Math. Comput., 147, 713-719 (2004) 